\title{
Methylation-dependent SOX9 expression mediates invasion in human melanoma cells and is a negative prognostic factor in advanced melanoma
}

\author{
Phil F Cheng ${ }^{1}$, Olga Shakhova ${ }^{3}$, Daniel S Widmer ${ }^{1}$, Ossia M Eichhoff ${ }^{1}$, Daniel Zingg ${ }^{3}$, Sandra C Frommel ${ }^{4}$, \\ Benedetta Belloni ${ }^{1}$, Marieke IG Raaijmakers ${ }^{1}$, Simone M Goldinger ${ }^{1}$, Raffaella Santoro ${ }^{4}$, Silvio Hemmi ${ }^{2}$, Lukas Sommer ${ }^{3}$, \\ Reinhard Dummer ${ }^{1}$ and Mitchell P Levesque ${ }^{1 *}$
}

\begin{abstract}
Background: Melanoma is the most fatal skin cancer displaying a high degree of molecular heterogeneity. Phenotype switching is a mechanism that contributes to melanoma heterogeneity by altering transcription profiles for the transition between states of proliferation/differentiation and invasion/stemness. As phenotype switching is reversible, epigenetic mechanisms, like DNA methylation, could contribute to the changes in gene expression.

Results: Integrative analysis of methylation and gene expression datasets of five proliferative and five invasion melanoma cell cultures reveal two distinct clusters. SOX9 is methylated and lowly expressed in the highly proliferative group. SOX9 overexpression results in decreased proliferation but increased invasion in vitro. In a B16 mouse model, sox9 overexpression increases the number of lung metastases. Transcriptional analysis of SOX9-overexpressing melanoma cells reveals enrichment in epithelial to mesenchymal transition (EMT) pathways. Survival analysis of The Cancer Genome Atlas melanoma dataset shows that metastatic patients with high expression levels of SOX9 have significantly worse survival rates. Additional survival analysis on the targets of SOX9 reveals that most SOX9 downregulated genes have survival benefit for metastatic patients.
\end{abstract}

Conclusions: Our genome-wide DNA methylation and gene expression study of 10 early passage melanoma cell cultures reveals two phenotypically distinct groups. One of the genes regulated by DNA methylation between the two groups is SOX9. SOX9 induces melanoma cell invasion and metastasis and decreases patient survival. A number of genes downregulated by SOX9 have a negative impact on patient survival. In conclusion, SOX9 is an important gene involved in melanoma invasion and negatively impacts melanoma patient survival.

\section{Background}

Melanoma is an aggressive skin cancer that originates from melanocytes, that is, pigment cells that reside in the basal layer of the epidermis and are derived from the neural crest during early development [1]. It is the most life-threatening neoplasm of the skin and is considered a major health problem due to rising incidence and mortality rates $[2,3]$. Melanoma is a tumor with a high degree of heterogeneity and this phenotypic heterogeneity is reversible [4-7]. In addition to being a challenge for basic research, melanoma plasticity is a major hurdle for

\footnotetext{
* Correspondence: mitchellpaul.levesque@usz.ch

'Department of Dermatology, Faculty of Medicine, University Hospital Zürich, and University of Zürich, Wagistrasse 14, CH-8952 Zürich, Switzerland Full list of author information is available at the end of the article
}

successful treatment [8]. Investigating the molecular basis of phenotypic heterogeneity is crucial to better understand melanoma progression and should provide useful insights for the development of more effective therapies.

In an effort to elucidate the molecular mechanisms of melanoma progression, significant differences have been detected between melanoma cells from the same lesion $[4,6,9]$. We and others have found that melanoma cells generally express two distinct gene expression signatures, that these signatures correlate with in vitro characteristics and these phenotypes are reversible depending on their cellular microenvironments [10-12]. One signature is characterized by the upregulation of several melanocytic genes like MITF, TYR, and DCT. These melanoma cells 
are highly proliferative and weakly invasive in vitro and so are named the proliferative phenotype. The other signature is characterized by the upregulation of many mesenchymal genes such as $W N T 5 A, T G F \beta$, and FGF2. In contrast to the proliferative cells, these cells are highly invasive but have a low proliferative capacity in vitro and are thus named the invasive phenotype. Meta-analysis of all available melanoma microarray datasets available on the NCBI GEO database confirmed these two gene signatures in $86 \%$ of the 536 melanomas [13]. Immunohistochemical analyses of MITF and WNT5A, markers of the proliferative and invasive phenotype, respectively, of human primary and metastatic melanomas displayed an anti-correlative staining pattern confirming that these phenotypes exist in vivo [14]. Together these findings culminated in the phenotype switching model for melanoma progression, in which melanoma cells respond to changing micro-environmental signals, such as hypoxia, by reprogramming their gene expression patterns to switch between states of proliferation and invasion $[9,15]$. Thus, phenotype switching has important implications in melanoma progression. Invasive phenotype cells characterized by low MITF expression, have stem-like properties [16], including the ability to initiate tumors with high efficiency [17]. Consequently, tumors comprise a mix of MITF positive and negative melanoma cells [18].

DNA methylation provides a stable and heritable gene regulatory mechanism for which melanoma cells could alter the expression of many genes [19]. Aberrant DNA methylation is a mechanism known to cause tumorigenesis [20]. Tumor suppressor genes become silenced by hypermethylation of their promoter region, thus promoting tumorigenesis. Global hypomethylation has been observed in many cancers, including melanoma, to decrease with progression of the disease [21-23]. DNMT3a and DNMT3b, the de novo DNA methyltransferases, were shown to have increased expression in metastatic melanomas compared to primary melanomas [24]. Another group showed that DNMT3a is required for melanoma development and metastasis in a melanoma mouse model [25]. Several signaling pathways have been shown to be deregulated as a result of aberrant DNMT-dependent methylation in melanoma, which include MAPK, WNT, PI3K, $\mathrm{pRB}$, and pathways in cell cycle, apoptosis, invasion, and metastasis [26]. Progressive global DNA hypomethylation has been observed in malignant melanocyte transformation, and surprisingly transformation was blocked in the presence of 5-Aza-2-deoxycytidine (decitabine, Aza), a DNMT inhibitor [22]. It would suggest that targeted hypomethylation is required for malignant transformation and not overall global hypomethylation caused by Aza treatment. This is supported by our observation that treating proliferative melanoma cells with Aza had no measureable effect on their invasive abilities (data not shown). 5-Aza-2-deoxycytidine treatment of various melanoma cell lines was shown to increase SOX9 expression and induce expression of p27 and p21 [27]. SOX9 is a transcription factor involved in neural crest specification [28] and SOX9 overexpression in melanoma cell lines have been shown to induce cell cycle arrest in a p21 dependent manner [29]. Taken together, it would suggest that DNA methylation has a crucial role in malignant transformation and progression by altering the landscape of the methylome to promote tumor progression, and SOX9 is one of the targets of DNA methylation that induces cell cycle arrest.

In this study, we examine the expression of the DNMTs between the proliferative and invasive melanoma cell cultures and describe the differential melanoma methylome by MeDIP-chip. We confirm that SOX9 expression is regulated by DNA methylation and has a role in cell cycle regulation, invasion in vitro and in vivo and could be a prognostic marker for overall survival in metastatic melanoma patients.

\section{Results \\ Proliferative melanoma cells have higher levels of global DNA-methylation}

We have previously established melanocytic markers like MLANA to distinguish between the proliferative and invasive phenotype on a cohort of primary melanoma cell cultures (Additional file 1: Figure S1) [11,30]. To investigate if methylation differences exist between the proliferative and invasive melanoma phenotypes, we compared five proliferative (M000921, M010817, M080423, M980513, and M050829) and five invasive (M990115, M010119, M080201, M080307, and M080310) melanoma cell cultures for expression of de novo DNA methyltransferases DNMT1, 3a, and 3b (Figure 1A). We observed that the invasive phenotype melanoma cell cultures had about 51.1\% less expression of DNMT1 as compared to the proliferative phenotype melanoma cell cultures (Figure 1A). DNMT3b had about 50\% less protein expression in the proliferative melanoma cell cultures compared to the invasive melanoma cell cultures (Figure 1A). However, DNMT3a was not differentially expressed between the proliferative and invasive phenotype (Figure 1A). Global methylation analysis by methyl-cytosine ELISA showed that the invasive phenotype cells have significantly less DNA methylation in their genome compared to the proliferative phenotype cells, $13.0 \%$ to $20.9 \%$, respectively (Figure 1B). This raises the possibility that differential methylation exists between the proliferative and invasive phenotype.

\section{A 73-gene signature is significantly differentially methylated and expressed in proliferative melanoma cells}

The difference in global methylation levels and protein expression of DNMT1 and DNMT3b prompted us to 


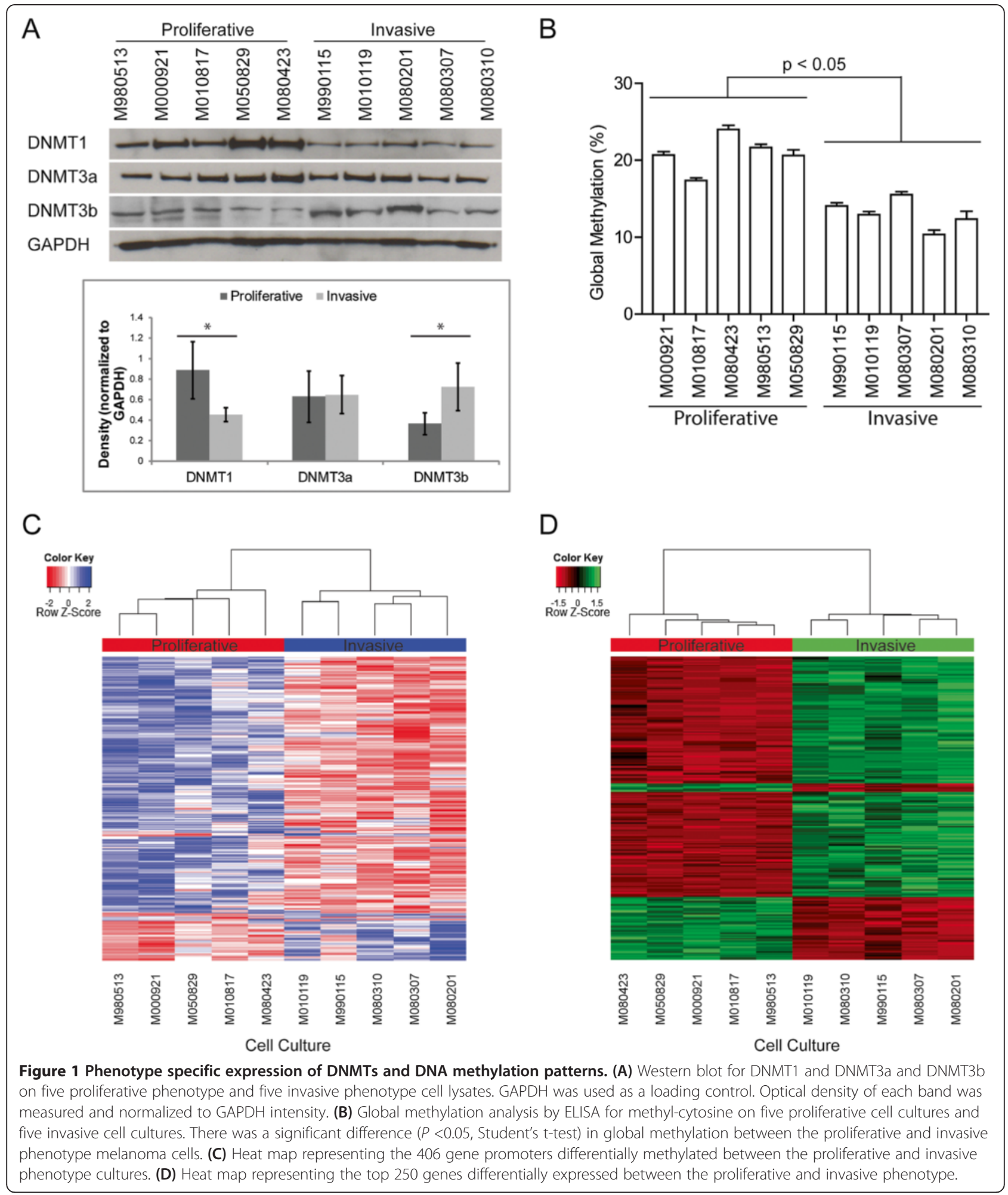

investigate the methylation profiles of the proliferative and invasive phenotype melanoma cells. To determine which CpG islands were differentially methylated between the proliferative and invasive phenotypes, we immunoprecipitated methylated DNA from five proliferative and five invasive melanoma cell cultures by MeDIP [31] followed by hybridization to Nimblegen Human DNA Methylation $3 x 720 \mathrm{~K}$ CpG Island Plus RefSeq Promoter Arrays. This array contains 720,000 probes for 22,532 promoter regions and 27,728 CpG islands. We calculated the differential 
methylation levels between the five proliferative and five invasive melanoma cells with a sliding window ANOVA test with the $\mathrm{R}$ package DMR supplied from Nimblegen. We found 406 gene promoters to be significantly and differentially methylated between the proliferative and invasive phenotypes (Figure 1C). A total of 320 promoter regions were hypermethylated in the proliferative phenotype and 86 promoter regions were hypermethylated in the invasive phenotype. The greater number of hypermethylated regions in the proliferative phenotype would be consistent with the global methylation data.

Gene expression data for the 10 melanoma cell cultures previously generated by us [13] were reanalyzed for differential gene expression between the proliferative and invasive phenotypes using the $\mathrm{R}$ package limma [32]. A total of 1,750 genes were differentially expressed between the proliferative and invasive phenotype (fold change $>2$, FDR corrected $P<0.05$ ) (Figure $1 \mathrm{D}$ ). We then analyzed the relationship between the promoter methylation status and mRNA expression levels for all genes in both datasets. Genes were filtered for a peak score $>2$ for methylation, fold change $>2$ for gene expression and an FDR-corrected $P$ value $<0.05$. A total of 73 genes showed both significant differential DNA methylation and significant differential expression between the proliferative and invasive phenotype (Additional file 2: Table S1). Sixty-two genes from the proliferative phenotype had hypermethylated promoters and low RNA expression and 11 genes in the invasive phenotype had hypermethylated promoters and low RNA expression as compared to the proliferative phenotype. This suggests that methylation has a role in regulating a portion of the genes differentially expressed between the proliferative and invasive phenotype. We hypothesized that the 73 genes with both differential DNA methylation and mRNA expression between the proliferative and invasive melanoma cells were likely to be true targets of epigenetic regulation in melanoma. To determine which groups of genes were functionally important, we performed pathway analysis of the 73 genes on MetaCore. We looked for enrichment of pathways under GO processes, process networks, and Pathway maps (Additional file 3: Table S2). Interestingly, we observed significant enrichment in pathways involved in EMT, melanoma, and cell differentiation. We decided to focus on SOX9 for validation due to its known function in melanocyte differentiation and melanoma progression $[29,33,34]$.

\section{Sox9 expression is silenced in proliferative melanoma cells through promoter DNA methylation}

From the methylation array, the area that had the most enrichment for methylation was about $2 \mathrm{~kb}$ upstream of the SOX9 transcriptional start site thus we validated the CpG island located there via sequencing of bisulfite-treated genomic DNA in the 10 melanoma cell cultures (Figure 2A). There are three predicted transcription factor binding sites in that upstream promoter region of $S O X 9$ for $M E F 2$, E2F, and $H N F 3 B$. We analyzed the DNA methylation status of a cluster of 17 CpGs across a 283-bp region and 15 CpGs across a 256-bp region of a CpG island, located approximately $-2,500$ bp to $-2,000$ bp upstream of the SOX9 transcriptional start site. The majority of $\mathrm{CpGs}$ in both regions of the SOX9 promoter were consistently methylated in the proliferative phenotype melanoma cell cultures and consistently unmethylated in the invasive phenotype melanoma cell cultures.

To confirm that promoter DNA hypermethylation correlated with transcriptional silencing of SOX9, we assessed mRNA levels using real-time RT-PCR in the 10 melanoma cell cultures. SOX9 mRNA was expressed robustly in the invasive phenotype melanoma cell cultures compared to the proliferative phenotype melanoma cell cultures $(P<0.05)$ (Figure 2B). Protein expression of SOX9 was detected in all invasive phenotype melanoma cell lysates, but little to no expression of $\mathrm{SOX} 9$ was seen in the proliferative phenotype melanoma cell lysates (Figure 2C). To validate that $S O X 9$ is indeed regulated by DNA methylation, we treated the five proliferative phenotype melanoma cell cultures with $5 \mu \mathrm{M} 5$-Aza-2'-deoxycytidine (a DNMT inhibitor) for $72 \mathrm{~h}$. Re-expression of SOX9 was detected by western blot (Figure 2D). Thus, SOX9 expression is regulated by DNA methylation between the proliferative and invasive phenotype.

\section{SOX9 mediates proliferation and invasion in melanoma cell cultures}

SOX9 is expressed in the invasive phenotype and we have previously described the greater invasive potential of invasive versus proliferative phenotype melanoma cells $[30,35]$. We hypothesized that some of the differentially expressed genes could have a role in generating this invasive capacity, thus we wanted to see if SOX9 would have a role in invasion. SOX9 was knocked down with siRNA, and then the invasive ability of two invasive phenotype melanoma cell cultures was measured: M080201 and M080310. Treatment with two independent siRNAs for SOX 9 achieved about 70\% knockdown of SOX9 mRNA in M080201 and M080310 (Figure 3A). The invasive capacity of M080201 and M080310 decreased significantly $(P<0.05)$ from $30 \%$ to $11.5 \%$ and from $64.8 \%$ to $36.6 \%$, respectively, after $48 \mathrm{~h}$ treatment with siRNA targeting SOX9 (Figure 3B and C). Proliferation was unaltered from SOX9 knockdown (data not shown).

Consistent with this observation, we overexpressed SOX9 in proliferative phenotype melanoma cell cultures (that is, M010817 and M980513) by lentiviral transfection (Figure 3D). Overexpression of SOX9 was previously shown to drive melanoma cells into cell cycle arrest [29]. 


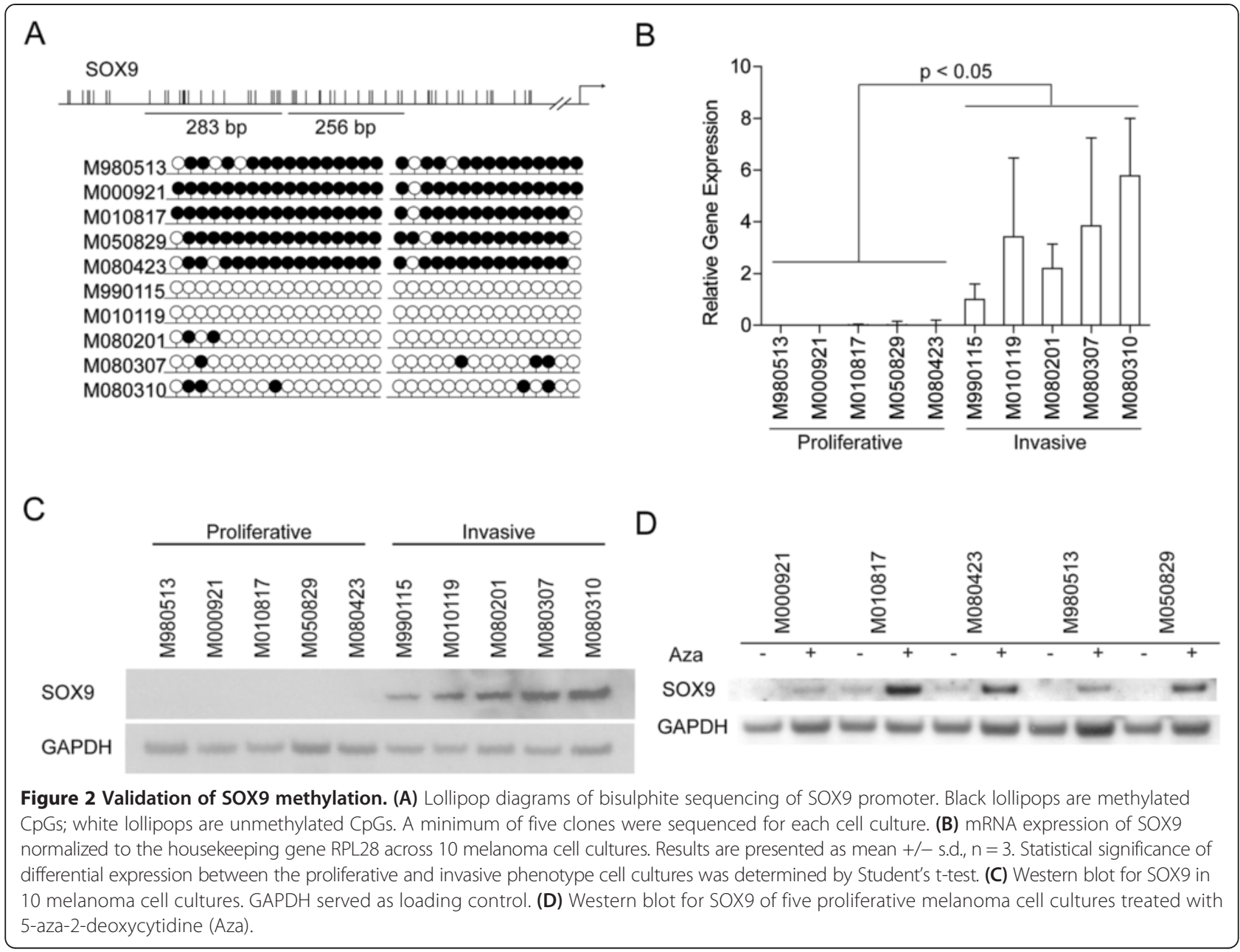

We measured proliferation and cell cycle progression by EdU and PI staining, respectively, and observed the cells transfected with vector have $50.5 \%$ in G1 phase, $37 \%$ in $\mathrm{S}$ phase, and $12.5 \%$ in $\mathrm{G} 2 / \mathrm{M}$ phase, whereas the cells overexpressing SOX9 have $64.8 \%$ in G1 phase, $17.7 \%$ in S phase, and $17.5 \%$ in $\mathrm{G} 2 / \mathrm{M}$ phase $(P<0.05)$ (Figure 3E and F). The invasive capacity of M010817 and M980513 were significantly increased from $0.95 \%$ to $9.0 \%(P<0.05)$ and from $0.67 \%$ to $4.8 \%(P<0.05)$ from SOX9 overexpression (Figure $3 \mathrm{G}$ and $\mathrm{H}$ ). In concordance with previously published data on SOX9 in melanoma, we also see G1/G0 arrest when SOX9 is overexpressed along with increased invasion.

\section{SOX9 induces a partial invasive phenotype in proliferative melanoma cells}

To determine the effect of SOX9 overexpression on the proliferative phenotype, we performed microarray analysis of M010817 cells overexpressing SOX9. We detected 643 genes downregulated at least two-fold and 450 genes upregulated at least two-fold ( $P<0.05$ (Figure 4A, Additional file 4: Table S3). We overlapped the gene signature from the SOX9 overexpression microarray with the gene signature from the 10 melanoma cell culture microarray to ask if SOX9 induced genes are enriched in the invasive phenotype. There were 98 genes that were upregulated and 55 genes that were downregulated in both the SOX9 overexpression and invasive phenotype gene sets. Hypergeometric distribution of the overlap of the SOX9 microarray with 10 melanoma cell culture array was significant $(P<0.001)$ (Figure $4 \mathrm{~B})$. Thus, SOX9 apparently regulates about $10 \%$ of the genes that define the invasive phenotype gene set. This suggests that SOX9 activation contributes to the invasive phenotype but other factors are also required for the full transition.

\section{In vivo function of SOX9 overexpression}

To examine the effects of SOX9 in vivo, we utilized the B16F1 mouse melanoma cells which do not express sox9 and are known not to metastasis in a tail vein injection assay. We transfected murine sox 9 transiently into the B16F1 cells and monitored its expression over $288 \mathrm{~h}$. Expression of sox 9 decreases over time but protein is still detectable at $288 \mathrm{~h}$ (Figure 5A). To assess the in vivo 

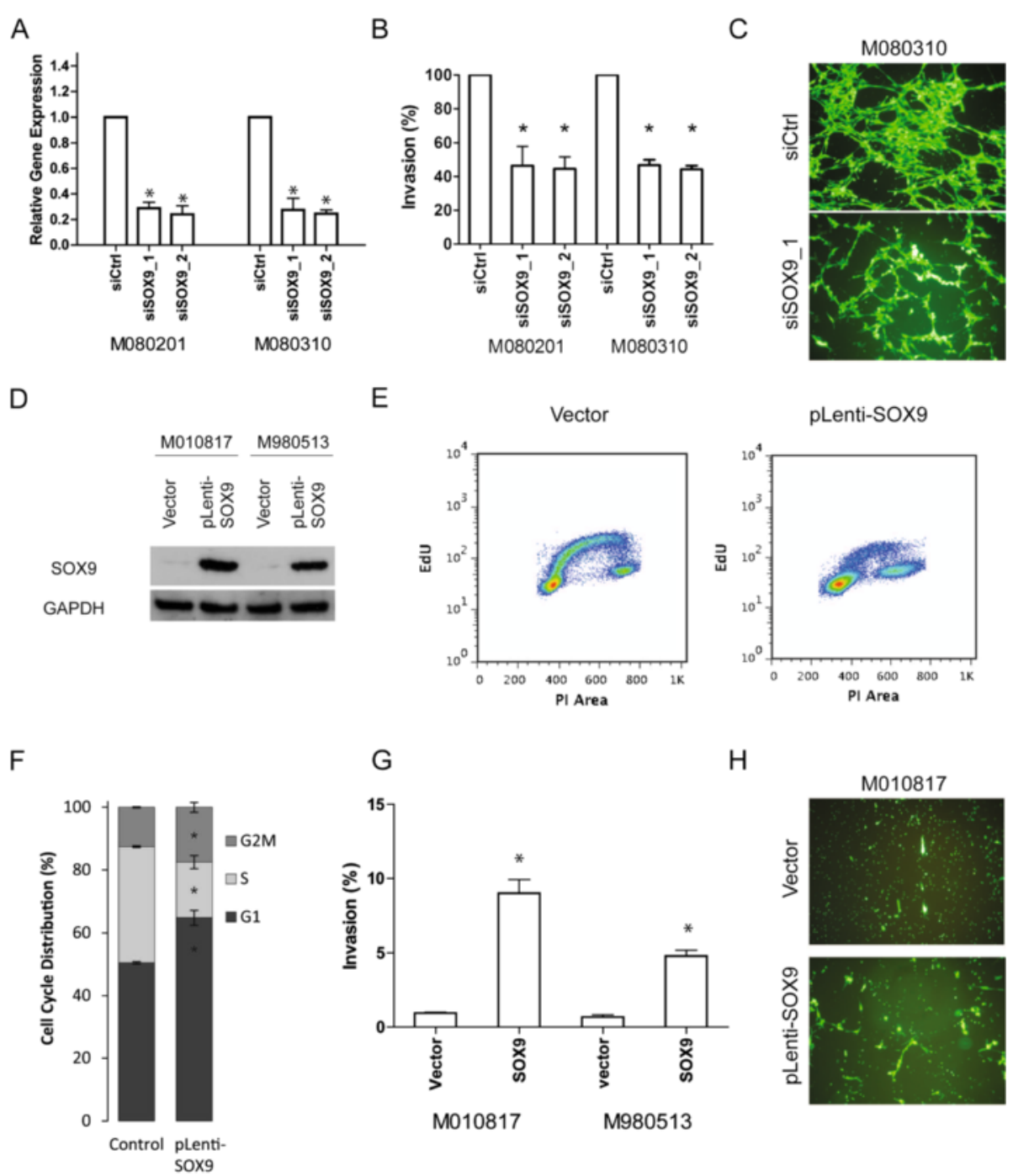

$\mathrm{H}$

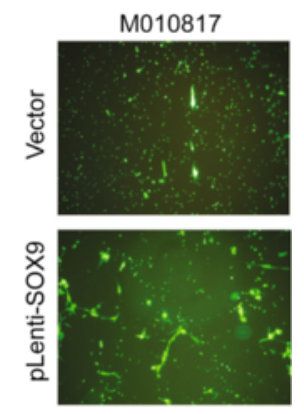

Figure 3 SOX9 mediates invasion and cell cycle arrest. (A) SOX9 knockdown by siRNA in two melanoma cell cultures M080201 and M080310. (B) Boyden chamber assay for siSOX9 knockdown in M080201 and M080310. (C) Representative picture of the Boyden chamber assay for knockdown of SOX9 in M080310. Top panel shows M080310 cells treated with control siRNA (siCtrl). Bottom panel shows M080310 cells treated with siSOX9_1. (D) SOX9 overexpression by lentiviral expression in proliferative phenotype melanoma cell cultures M010817 and M980513. (E) Proliferation assay by EdU pulse in cell cultures transfected with empty vector and pLenti-SOX9. (F) Cell cycle analysis by PI staining for cell cultures transfected with empty vector and pLenti-SOX9 (top and bottom left panels). Quantitation of cell cycle analysis ( ${ }^{*}=P<0.05$, right panel). (G) Boyden chamber assay for SOX9 overexpressing melanoma cell cultures M010817 and M980513 $\left(^{*}=P<0.05\right)$. (H) Representative picture of the Boyden chamber assay for SOX9 overexpression in M010817. Top panel shows M010817 transfected with empty vector. Bottom panel shows M010817 transfected with SOX9.

metastatic potential of sox 9 , C57BL/6 J mice were intravenously injected with B16F1 cells transfected with sox9 and empty vector (Figure 5B). Twelve days after injection, the mice were sacrificed and the lungs were analyzed for tumor nodules. B16F1 cells expressing sox9 had significantly more metastases compared to control, $P<0.05$.

\section{Validation with TCGA melanoma dataset}

The Cancer Genome Atlas (TCGA) has a melanoma dataset available for public access which contains over 300 tissue samples with RNAseq, DNA methylation, and clinical data. To validate our claim that SOX9 is regulated by DNA methylation, we performed a correlation analysis of SOX9 expression to SOX9 promoter methylation using the data from the TCGA database. Three consecutive probes cg10471574, cg21049501, and cg06234051 in the SOX9 promoter region have an anti-correlative association with the expression of $S O X 9$, $\mathrm{r}=-0.58,-0.61$, and -0.71 respectively (Figure $6 \mathrm{~A}$ ). Since high DNA methylation of SOX9 is correlated with low SOX9 expression it provides strong evidence that DNA methylation regulates $\mathrm{SOX} 9$ expression in vivo.

The melanoma dataset contains 68 primary samples and 268 metastatic samples. The 268 metastatic samples 


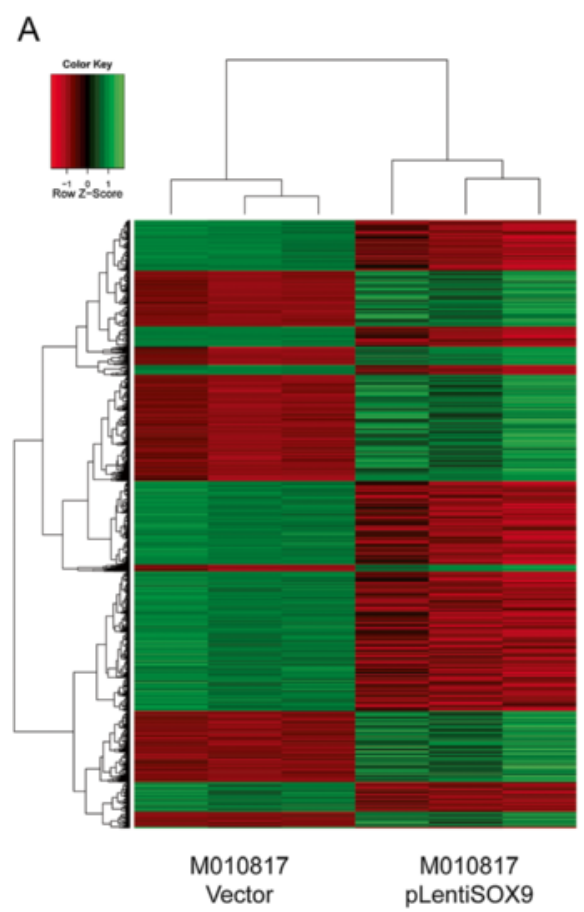

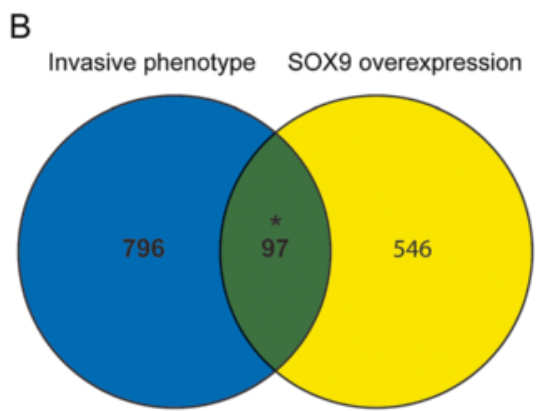

Up-regulated genes

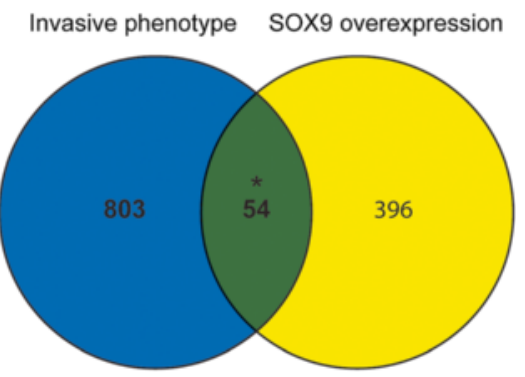

Down-regulated genes

Figure 4 Microarray analysis of SOX9 overexpression. (A) Heat map of M010817 cells overexpressing SOX9. (B) Overlap of upregulated genes and downregulated genes between the SOX9 microarray and 10 melanoma cell culture microarray.

are comprised with 172 lymph node tumors, 59 regional cutaneous or subcutaneous metastases, and 37 distant metastases. Due to the diversity of this dataset we analyzed the primary and metastatic samples individually. We segregated the population into thirds by SOX9 expression. We compared the upper and lower thirds for our analysis labelling them $S O X 9$ high and $S O X 9$ low. In both primary and metastatic datasets, the SOX9 high group had at least three times more expression than the SOX9 low group. We interrogated clinically relevant factors such as TNM staging, age, gender, and tumor type between the SOX9 high and SOX9 low group in the primary and metastatic datasets (Table 1, Additional file 5 : Table S4). All parameters were statistically insignificant as tested by the Chi-squared test and t-test for age in the primary melanoma dataset. Only two clinical parameters were significant in the metastatic dataset. T1 was significant 2 vs. $11(P=0.013)$ in the SOX9 low versus SOX9 high, respectively. N0 was also significant 26 vs. 44 $(P=0.031)$ in the SOX9 low versus SOX9 high, respectively.

We next tested if the genes differentially expressed between the SOX9 high and SOX9 low patients in the primary and metastatic datasets were the same in our SOX9 overexpression microarray. A total of 21 genes were differentially expressed between the SOX9 high and SOX9 low groups in the primary dataset with a minimum fold change of 2 and FDR corrected $P$ value $<0.05$ (Additional file 6: Table S5). No genes from this set overlapped with the SOX9 microarray. A total of 427 genes were differentially expressed between the SOX9 high and SOX9 low groups in the metastatic dataset with a minimum fold change of 2 and FDR corrected $P$ value $<0.05$ (Figure 6B, Additional file 7: Table S6). A total of 31 genes overlapped with the SOX9 microarray. Although the overlap was small, hypergeometric distribution of this overlap was significant $(P<0.05)$. To examine the pathways in which, SOX9 might play a role in vivo, we performed pathway analysis on the 427 genes. We saw significant enrichment of many EMT pathway processes such as 'Regulation of epithelial-to-mesenchymal transition (EMT)', 'TGF-beta dependent induction of EMT via SMADs', and 'Melanocyte development and pigmentation' (Figure 7A).

We interrogated if SOX9 has a role in overall patient survival in all patients regardless of primary or metastatic disease. There was a significant difference in 10-year survival rates between the SOX9 high and SOX9 low groups. SOX9 high patients had a median survival rate of 3.9 years whereas the SOX9 low patients had a median survival time of 5.8 years $(P<0.05)$ (Figure 7B). Cox multivariate analysis was carried out to identify if age, gender, TNM stage, and tumor type were significant contributing factors for 10-year survival of SOX9 high and SOX9 low patients. SOX9 expression (hazard ratio 2.343; 95\% confidence interval (CI) 1.402-3.915; $P=0.001$; SOX9 


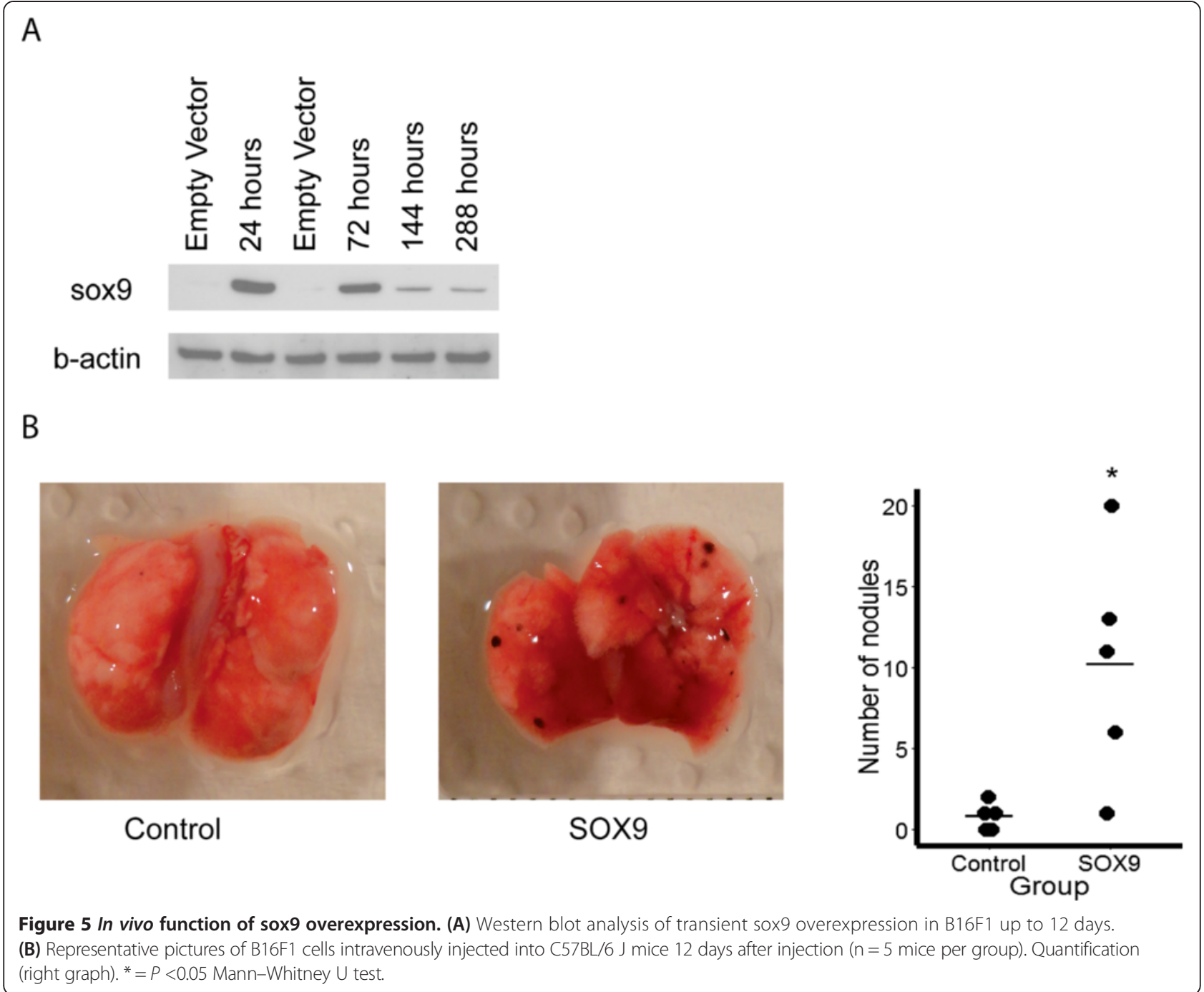

high vs. SOX9 low) and T4 stage (hazard ratio 2.145; 95\% CI 1.01-4.557; $P=0.047$; T4 vs. T0) were significant (Table 2). We also segregated the patients into primary, lymph node, metastasis and regional/distant metastasis and reassessed survival based on SOX9 expression. We saw that SOX9 expression in primary tumors had no effect on patient survival. However, the cohort of patients with high SOX9 expression in lymph nodes had significantly lower survival $(P=0.03)$, as in the regional/ distant metastasis cohort $(P=0.01)$ (Figure 7C).

We tested all 31 overlapping genes for survival benefit in the metastatic dataset (Additional file 8: Table S7). Twelve of the genes of this set were upregulated and 19 were downregulated when SOX9 expression was high. Most of the genes, 92\% (11/12), that were upregulated when SOX9 expression is high had no influence on patient survival, only one gene $7 \%(1 / 12)$ was associated with improved survival (Figure 7D). This suggests that the genes upregulated by SOX9 are not direct factors for patient survival. Interestingly, 58\% (11/19) of the genes downregulated when SOX9 expression is high were associated with improved survival and the other $42 \%$ (8/19) had no influence on patient survival (Figure 7D). This suggests that SOX9 represses a group of genes important for patient survival. Taken together, high SOX9 expression leads to poor survival possibly due to the downregulation of several genes that influence patient survival.

\section{SOX9 binds to the promoter regions of its target genes}

Using the SOX9 binding motif to screen for potential binding sites on the promoter regions from the 31 overlapping targets of the SOX9 microarray and the TCGA melanoma dataset, we found 19 of the 31 genes had a potential SOX9 binding site within a $3 \mathrm{~kb}$ region upstream from the TSS. To determine whether SOX9 directly binds to the promoter regions of these genes, we performed ChIP analysis using SOX9 antibodies on 


\section{A}

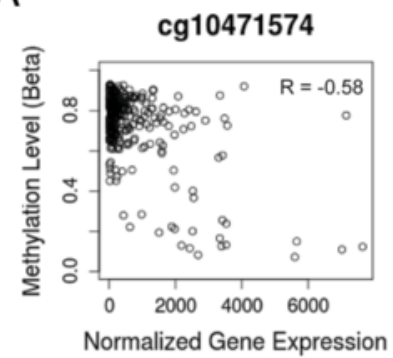

B

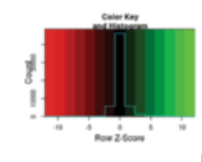

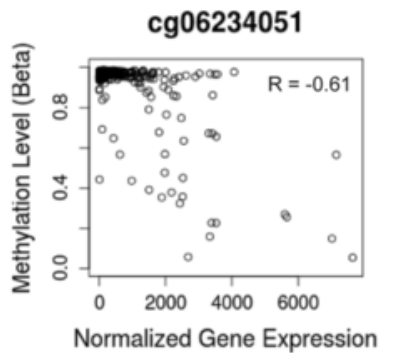

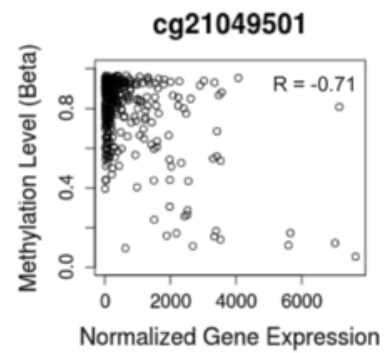

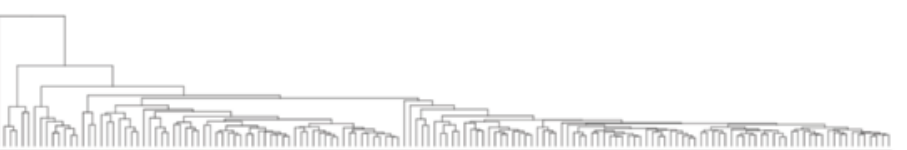

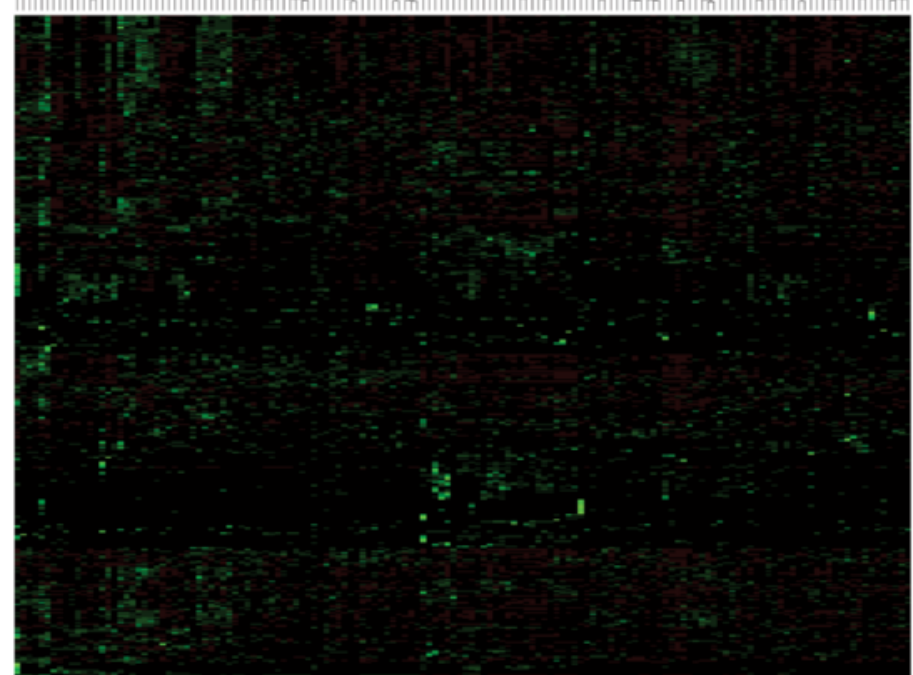

Figure 6 Validation of SOX9 methylation in TCGA and correlation with clinical features. (A) Correlation plots of RNAseq reads to b-values of methylation for SOX9. Three methylation probes of SOX9 are shown here to have significant anti-correlation of gene expression and DNA methylation. (B) Heatmap of the 427 genes differentially expressed between the SOX9 high and SOX9 low metastatic melanoma samples.

M010817-SOX9 cells and measured SOX9 occupancy at promoter regions of TMEM158, TBX3, and FYB, for which we could design specific primers for qPCR (Figure 7E). The specificity of this assay was demonstrated by the enrichment of three known SOX9 target sequences, COL2A1 intron 1 [36], p21 [29], and SOX10 [37]), as compared to a non-target gene (IP10). We observed a specific association of SOX9 with TMEM158, TBX3, and FYB, suggesting that TMEM158, TBX3, and FYB are direct targets of SOX9 in melanoma.

\section{Discussion}

Phenotypic, genetic, and epigenetic heterogeneity is a common feature in human melanomas $[4,5,14,38]$. Tumor subpopulations can be transient and have been seen to switch between phenotypic states in vivo $[4,6,9,12,39]$. We have previously described two subpopulations in melanoma, the proliferative phenotype and the invasive phenotype, which are defined by specific gene signatures, in vitro characteristics, and response to drug treatment $[11,13,30,35,40]$. Briefly, proliferative phenotype melanoma cells are distinguished by a high proliferative capacity and low invasive capacity and the invasive phenotype melanoma cells are distinguished by a low proliferative capacity and high invasive capacity. In this study, we found specific DNA methylation signatures for the proliferative and invasive melanoma phenotypes. We observed the invasive phenotype melanoma cell cultures had modest decrease of $5 \%$ in global methylation compared to the proliferative phenotype melanoma cell cultures. This may be due to decreased DNMT1 protein expression in the invasive phenotype melanoma cells. Global methylation levels have been observed to decrease as a cancerous lesion progresses from a benign tumor to metastasis $[22,41]$, and we observed our invasive phenotype melanoma cell cultures had decreased DNA methylation levels and were more invasive than the proliferative phenotype melanoma cell cultures, suggesting the invasive cell 


\begin{tabular}{|c|c|c|c|}
\hline Clinical parameter & SOX9 low & SOX9 high & $p$ value \\
\hline T0 & 15 & 13 & 0.706 \\
\hline T1 & 2 & 11 & 0.013 \\
\hline T2 & 17 & 17 & 1 \\
\hline T3 & 20 & 13 & 0.223 \\
\hline $\mathrm{T} 4$ & 16 & 16 & 1 \\
\hline No & 26 & 44 & 0.031 \\
\hline N1 & 16 & 9 & 0.162 \\
\hline N2 & 15 & 9 & 0.221 \\
\hline N3 & 12 & 9 & 0.513 \\
\hline NX & 1 & 0 & 1 \\
\hline MO & 67 & 64 & 0.793 \\
\hline M1 & 2 & 6 & 0.157 \\
\hline Female & 29 & 25 & 0.586 \\
\hline Male & 45 & 49 & 0.68 \\
\hline Lymph node & 48 & 49 & 0.919 \\
\hline $\begin{array}{l}\text { Regional cutaneous or } \\
\text { subcutaneous metastasis }\end{array}$ & 17 & 14 & 0.59 \\
\hline Distant metastasis & 9 & 11 & 0.655 \\
\hline Age & 57 & 59.9 & $0.213^{\mathrm{a}}$ \\
\hline
\end{tabular}

TNM stage, gender, and tumor location between the SOX9 high and SOX9 low patients were evaluated by the Chi-squared test.

${ }^{\mathrm{a}} \mathrm{Age}$ was evaluated by Student's t-test.

${ }^{*} P<0.05$.

cultures have progressed further in malignancy. Differential expression of the de novo DNA methyltransferase DNMT3b was also seen between the proliferative and invasive phenotype. These data are consistent with a model in which DNMT1 and DNMT3b have phenotype specificity and contribute to transcriptional heterogeneity by altering the methylation landscape of a melanoma cell in the context of melanoma phenotype switching. Pathway analysis of the 73 gene signature from the DNA methylation and gene expression array lead to the discovery of many transcription networks involved in development. These transcription factors were found to be hypomethylated and highly expressed in the invasive phenotype, which would suggest the invasive melanoma cell cultures may revert to a dedifferentiated state.

A number of other studies that have looked at genomewide DNA methylation in melanoma have indicated that several tumor suppressors are silenced by DNA methylation compared to normal melanocytes [42-44] and compared to benign nevi [45]. Also, a recent study investigating 5-hydroxymethylation $(5-\mathrm{hmC})$ in melanoma found a global decrease of 5 -hmC was necessary for melanoma formation [46]. The results from these studies indicate aberrant DNA methylation is an important process in melanoma development and progression. In our work, we looked at the differences in DNA methylation landscape between 10 primary melanoma cell cultures and uncovered two distinct populations, as previously demonstrated by gene expression microarray analysis from our group [13]. Surprisingly, the targets we found to be differentially methylated between the two phenotypes do not overlap with the targets found to be differentially methylated between normal melanocytes and melanoma, and benign nevi and melanoma. We did not detect any differential methylation in validated methylation gene sets such as COL1A2, NPM2, HSPB6, DDIT4L, and MTIG from Koga et al. [43] or UCHL1, COL1A2, THBS1, and TNFRSF10D from Bonazzi et al. [42]. As those studies were comparing the methylation state of normal melanocytes to melanoma and in this study we compare within melanoma phenotypes, this might indicate that a different set of pathways are activated or silenced by DNA methylation in melanoma progression compared to melanoma initiation. In either case, it is clear that epigenetic modifications such as DNA methylation play an important role in melanoma initiation as well as progression, and embryonic developmental program reactivation may be one of the critical outcomes of this modulatory activity.

In our study, a subset of our melanoma cell cultures had lower SOX9 expression due to a hypermethylated promoter and the other subset with high SOX9 expression had a hypomethylated promoter. We confirmed that SOX9 is regulated by DNA methylation by treating low SOX9 expressing cells with 5-aza-2-deoxycytidine treatment and saw re-expression of SOX9. To determine if the regulation of SOX9 by DNA methylation is a common mechanism in melanoma or just seen within our melanoma cell cultures, we interrogated the melanoma TCGA dataset for SOX9 and found that SOX9 gene expression and DNA methylation are anti-correlated at three consecutive methylation probes in 293 samples. This provides strong evidence that specific DNA methylation is the molecular mechanism that regulates SOX9 expression in melanoma. Alcazar et al. demonstrated that after decitabine treatment of A375 and B16 melanoma cells, the promoter of SOX9 becomes hypomethylated and SOX9 is re-expressed with induction of p27 and p21 for cell cycle arrest [27]. Passeron et al. also observed that SOX9 was downregulated in some melanoma cell lines and induction of SOX9 expression in these melanoma cell lines resulted in lower proliferation due to upregulation of p21 [29]. We also overexpressed SOX9 in low SOX9 melanoma cell cultures and observed G1/S cell arrest, which is consistent with the study from Passeron et al. Although the proliferation rate is reduced, the invasive capacity of these SOX9 overexpressing cells is increased, which phenocopies the endogenous SOX9 expressing 
A

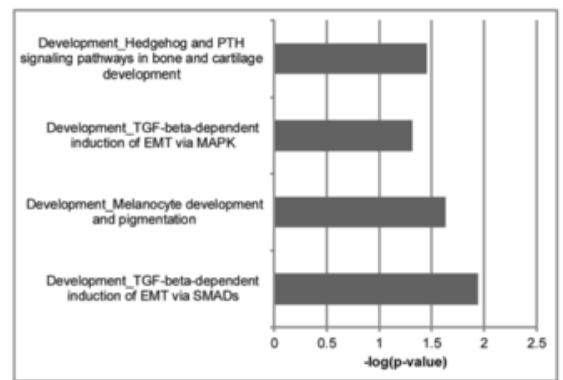

B

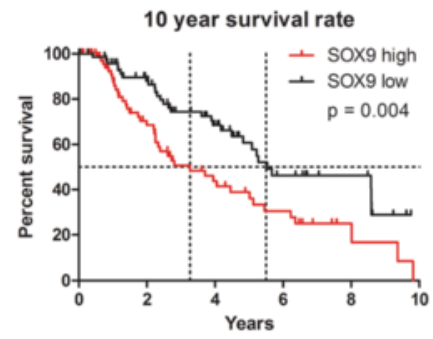

C
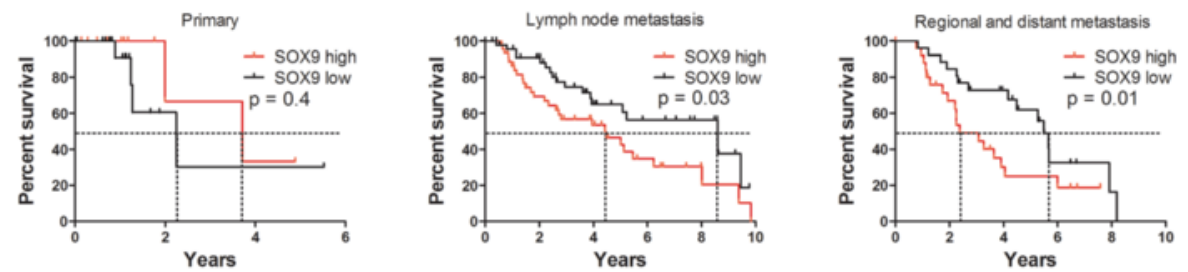

D

Genes upregulated by SOX 9

Genes downregulated by SOX 9

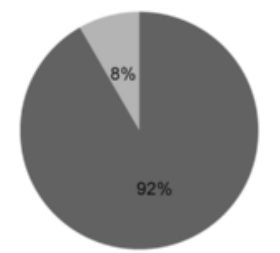

- No effect on survival = Improved survival

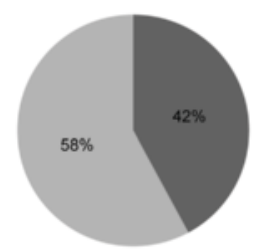

- No effect on survival = Improved survival

E

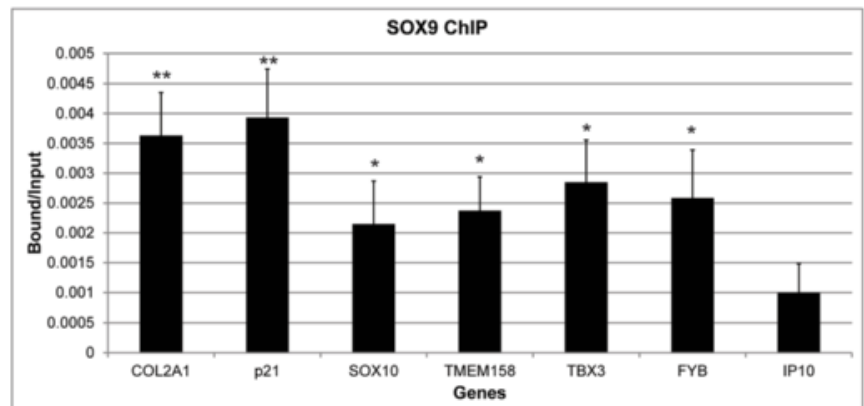

Figure 7 TCGA analysis of SOX9. (A) Pathway analysis from MetaCore for SOX9 reveals many pathways involved in EMT. (B) Median survival time for patients with high SOX9 expression is 3.9 years $(n=74)$. Median survival time for patients with low SOX9 expression is 5.8 years $(n=74)$. The difference in survival is significant $(P<0.05)$. (C) Survival analysis for high and low SOX9 expression segregated into primary, lymph node, and metastatic cohorts. (D) Pie charts displaying the number of SOX9 target genes that have a contribution to patient survival. (E) ChIP analysis for SOX9 binding targets. Enrichment of the promoter regions for TMEM158, TBX3, and FYB were similar to positive controls COL2A1, p21, and SOX10 and greater than negative control IP10. Data are shown as bound vs. input. Error bars indicate standard error of the mean of three independent experiments. IgG controls not shown on graph because they were below detection limit.

cells. Conversely, knockdown of SOX9 in the invasive phenotype melanoma cells reduced the invasive capacity of the cells. Microarray analysis of SOX9 overexpression revealed an EMT-like transcriptional signature and had $10 \%$ overlap with invasive phenotype gene signature which supports the notion that SOX9 is a factor that contributes to the invasive phenotype. In vivo, sox 9 expression in
B16F1 cells increases their metastatic potential causing more tumor lung nodules in the tail vein injection assay. Taken together, SOX9 is a gene that is regulated by DNA methylation and functionally, SOX9 mediates cell cycle progression, invasion, and metastasis in melanoma.

TCGA is a great resource for clinical and nextgeneration sequencing data on human tumors. We 
Table 2 Multivariate cox regression on metastatic melanoma patients

\begin{tabular}{|c|c|c|c|c|}
\hline Covariate & HR & Lower $95 \%$ & Upper $95 \%$ & $\mathrm{p}$-value \\
\hline $\begin{array}{l}\text { SOX9 }(\text { SOX9 low =0, } \\
\text { SOX9 high }=1)\end{array}$ & 2.343 & 1.402 & 3.915 & $0.001^{* * *}$ \\
\hline Age & 0.995 & 0.977 & 1.103 & 0.59 \\
\hline $\begin{array}{l}\text { Gender (female =0, } \\
\text { male =1) }\end{array}$ & 0.756 & 0.457 & 1.25 & 0.276 \\
\hline \multicolumn{5}{|l|}{ TO (used as reference) } \\
\hline $\mathrm{T} 1$ & 0.431 & 0.117 & 1.598 & 0.208 \\
\hline $\mathrm{T} 2$ & 0.943 & 0.416 & 2.138 & 0.888 \\
\hline T3 & 1.022 & 0.489 & 2.132 & 0.955 \\
\hline T4 & 2.145 & 1.01 & 4.557 & $0.047^{*}$ \\
\hline \multicolumn{5}{|l|}{ No (used as reference) } \\
\hline N1 & 1.673 & 0.813 & 3.446 & 0.162 \\
\hline N2 & 1.365 & 0.673 & 2.771 & 0.388 \\
\hline N3 & 1.322 & 0.574 & 3.044 & 0.512 \\
\hline \multicolumn{5}{|l|}{ MO (used as reference) } \\
\hline M1 & 2.023 & 0.672 & 6.09 & 0.21 \\
\hline \multicolumn{5}{|l|}{$\begin{array}{l}\text { Lymph Node (used as } \\
\text { reference) }\end{array}$} \\
\hline $\begin{array}{l}\text { Regional Cutaneous or } \\
\text { Subcutaneous Metastasis }\end{array}$ & 0.844 & 0.452 & 1.577 & 0.595 \\
\hline Distant Metastasis & 1.83 & 0.957 & 3.499 & 0.068 \\
\hline
\end{tabular}

SOX9 expression, age, gender, TNM stage, and tumor location were evaluated in a multivariate cox regression model. Hazard ratio (HR) and the $95 \% \mathrm{Cls}$ are displayed for each covariate.

${ }^{*} P<0.05$.

***P $P<0.001$.

took advantage of the melanoma dataset and demonstrated that SOX9 expression levels have a significant impact on survival of metastatic melanoma patients but SOX9 did not have a significant impact on survival in patients with primary melanomas. This could suggest that SOX9 is required for progression of primary melanoma into metastasis and metastatic tumors with high SOX9 are more aggressive to the patient. There were no clinical metrics that could distinguish SOX9 high or low in primary melanoma. Only T1 and N0 stage in metastatic melanomas were significant between SOX9 high and low. Survival analysis of metastatic patients with high SOX9 expression versus low SOX9 expression revealed a significant difference in the overall 10-year survival rates. Patients with highSOX9 expressing tumors had a 2.3 times increased risk of death compared to patients with low SOX9 expressing tumors. Based on these findings and the invasive properties of high SOX9 expressing melanomas, it would suggest that SOX9 expression in melanomas could push the tumor toward more aggressive metastasis. Thus, SOX9 could potentially be a prognostic marker for metastatic melanoma.
We performed differential gene expression analysis on the RNAseq dataset where we defined the SOX9 high group as having a minimum of three-fold greater expression than the SOX9 low group. We only saw an overlap of 31 genes between both datasets; however, the overlap was significant as determined by hypergeometric distribution. The contribution of heterogeneity in the melanoma TCGA patient population would be one of the largest factors for the difference in gene signatures between our SOX9 microarray and TCGA RNAseq data. Nonetheless, the significant overlap of genes narrow down the potential targets of SOX9. To confirm that the targets of SOX9 have prognostic value for the patients, we performed survival analysis on all 31 genes. Surprisingly, 58\% (11/19) of the genes downregulated by SOX9 were associated with improved survival, which strongly suggests SOX9 represses a set of genes that decrease tumor malignancy. Genes that were upregulated by SOX9 expression had little impact on patient survival which implies that SOX9 expression alone is sufficient to drive disease progression. From this list of 31 genes, 19 of them had a potential SOX9 binding site in its promoter. We could validate TMEM158, TBX3, and FYB as direct targets of SOX9 binding by chromatin immunoprecipitation. FYB is downregulated when SOX9 levels are high suggesting a repressive effect of SOX9 on this gene. FYB is required for inflammatory cytokine production [47] but no known link has been established with melanoma. TMEM158 is upregulated by SOX9 but no clear role has been established for the gene in melanoma. TBX3 is also upregulated by SOX9 and TBX3 is known to cause increased invasiveness in melanoma $[48,49]$, suggesting TBX3 could be an effector gene that drives the invasive phenotype we see in SOX9 high cells and in patients.

\section{Conclusion}

In conclusion, we found SOX9 to be regulated by DNA methylation, and high SOX9 expression leads to poor survival in melanoma patients due to the activation of EMT-like genes and the downregulation of potential tumor suppressor genes in melanoma cells. This was confirmed in vivo, and new direct targets of SOX9 that may mediate its function in tumor progression were identified by transcriptional profiling and chromatin-immunoprecipitation. Future therapies targeting SOX9 could be beneficial for patients to prevent progression and especially when combined with therapies targeting cells of the proliferative phenotype. Further investigation would be required to determine if SOX9 would have early prognostic value for tumor malignancy. 


\section{Materials and methods Cell culture}

Melanoma cell cultures were established from surplus material from primary cutaneous melanoma and melanoma metastases removed by surgery [50]. Written informed consent was approved by the local IRB (EK647 and EK800). Clinical diagnosis was confirmed by histology and immunohistochemistry. Melanoma cells were released from tissue biopsies and grown as previously described [51]. Melanoma cell cultures were maintained in RPMI (Invitrogen, Carlsbad, CA, USA) supplemented with $5 \mathrm{mM}$ glutamine, $1 \mathrm{mM}$ sodium pyruvate, and $10 \%$ heat-inactivated fetal calf serum, and cultured at $37^{\circ} \mathrm{C}$ and $5 \% \mathrm{CO}_{2}$. As RNA was extract previously from these cell cultures for gene expression array analysis, all cell cultures used for experiments in this paper were within five passages of the RNA isolation time point.

\section{5-methylcytosine relative content analyses}

Global DNA methylation level was evaluated by MethylFlash Methylated DNA Quantification Kit (Epigentek, Farmingdale, NY, USA) as per manufacturer's instructions.

\section{MeDIP assay and analysis}

The MeDIP assay was performed as described [31]. A monoclonal antibody to 5-Methylcytidine (BI-MECY100, Eurogentec, Belgium) was used for immunoprecipitation. The immunoprecipated DNA and sonicated input DNA were differentially labeled with fluorescent dyes (Cy3 and $\mathrm{Cy} 5$, respectively) and hybridized to Human DNA Methylation 3x720K CpG Island Plus RefSeq Promoter Arrays (Roche Nimblegen, Madison, WI, USA). Acquisition and analysis was performed using Nimblescan 2.5 and $\mathrm{R}$ package DMR provided by Nimblegen. All data have been deposited into NCBI GEO GSE57971.

\section{Gene expression analysis}

Gene expression datasets were obtained from NCBI GEO GSE33728 [13], and analysis was performed by $\mathrm{R}$ using the limma package. $P$ values were adjusted by FDR multiple hypothesis test correction.

\section{Bisulphite sequencing}

Genomic DNA was extracted from primary melanoma cell cultures and subjected to bisulfite (BS) modification (EZ DNA Methylation Gold Kit, Zymo Research, Irvine, CA, USA). To validate the DNA methylation status of individual DNA molecules, we cloned bisulfite-converted PCR fragments into the pCR2.1 vector using the TOPO-TA cloning kit (Invitrogen, Carlsbad, CA, USA). Individual colonies were screened for the insert, and the region of interest was sequenced using M13 primers. A minimum of five clones were sequenced for each region of interest. Lollipop diagrams were generated using BiQ Analyzer [52]. Primers used for bisulphite PCR are shown in Table 3.

\section{Treatment with decitabine}

Decitabine (5-Aza-2'-deoxycytidine, Sigma Chemical (Aza)) was dissolved in DMSO as a $10 \mathrm{mM}$ stock solution, aliquoted, and kept at $-20^{\circ} \mathrm{C}$. Primary melanoma cell cultures were seeded in Petri dishes (approximately 5,000 cells $/ \mathrm{cm}^{2}$ ) in RPMI untreated or treated with Aza $(5 \mu \mathrm{M})$ for $72 \mathrm{~h}$, with fresh drug-supplemented medium every $24 \mathrm{~h}$.

\section{mRNA expression analysis}

Total RNA was isolated using Trizol according to manufacturer's instructions (Invitrogen). In total, $1 \mu \mathrm{g}$ aliquots of RNA were reverse transcribed with Reverse Transcription System (Promega) according to the manufacturer's instructions. Data collection and analysis were performed by ABI Viia7 Fast Real-Time PCR Systems (Applied Biosystems). Gene expression values of averaged triplicate reactions were normalized to RPL28 expression levels. RPL28 primers are as follows: 5'-GCAATTGG TTCCGCTACAAC-3' and 5'-TGTTCTTGCGGATCAT GTGT-3'. The primers for RT-PCR were purchased from QIAGEN: SOX9 (Hs_SOX9_1_SG).

\section{Western blot}

Cells were washed twice with cold phosphate-buffered saline (PBS) and lysed at $4^{\circ} \mathrm{C}$ in lysis buffer containing $20 \mathrm{mM}$ Tris- $\mathrm{HCl}$ (pH 7.5), 1\% Triton X-100 (SigmaAldrich, St Louis, MO, USA), $137 \mathrm{mM} \mathrm{NaCl}, 10 \%$ glycerol, and protease and phosphatase inhibitors (Roche, Basel, Switzerland). Proteins were separated by SDS-PAGE using the NuPAGE SDS-PAGE Gel System (Invitrogen) under reducing conditions. A total of $15 \mu \mathrm{g}$ of protein was mixed with $9 \mu \mathrm{L}$ of NuPage LDS sample buffer $(4 \times)$ (Invitrogen, NP0007), 3.6 $\mu \mathrm{L}$ of NUPAGE Sample Reducing (Invitrogen, NP0009) and filled up to $36 \mu \mathrm{L}$ with RIPA buffer. This mixture was incubated at $85^{\circ} \mathrm{C}$ for $10 \mathrm{~min}$ while shaking at $900 \mathrm{rpm}$. Samples were loaded on NuPage precast gels (Invitrogen). Membranes were probed with the following antibodies: SOX9 (GTX109661, GeneTex, Hsinchu City, Taiwan); DNMT1 (ab13537, Abcam, Cambridge, UK); DNMT3a (ab2850, Abcam, Cambridge, UK); DNMT3b (ab16049,

Table 3 Primers for bisulphite sequencing

\begin{tabular}{lll}
\hline Gene & Primer & Tm \\
\hline SOX9_1 & F: 5'-GGATTGGGGTTTTTATTTTT-3' & $59^{\circ} \mathrm{C}$ \\
& R: 5'-TTCAATTTCTTCCCTTTCCT-3' & \\
SOX9_2 & F: 5'-AGGTTATTAGGGTAGATTGGAGG-3' & $59^{\circ} \mathrm{C}$ \\
& R: 5'5AAATACATATCCCATCACAACC-3' & \\
\hline
\end{tabular}


Abcam, Cambridge, UK); GAPDH (ab9483, Abcam, Cambridge, UK);

\section{siRNA knockdown}

Silencing RNA (siRNA) transfection of melanoma cells was carried out using INTERFERin transfection solution according to the manufacturer's protocol (Polyplustransfection, Illkirch, France). Cells were transfected with $5 \mathrm{nM}$ of siRNA (Qiagen) for $72 \mathrm{~h}$ before RNA or protein was extracted. As control siRNA, the All-Star negative siRNA sequence (Qiagen) was used, and genespecific siRNAs targeting siSOX9 (SI00007595, SI00007609) were obtained from Qiagen.

\section{SOX9 lentiviral transfection}

Lentiviral particles containing plasmids expressing fulllength SOX9 cDNA or eGFP were transfected into melanoma cells for $48 \mathrm{~h}$. Media supplemented with $4 \mathrm{ng} / \mathrm{mL}$ blasticidin was used for selection. After 1 week of selection, protein lysate was extracted and analyzed for SOX9 expression. Plasmids for eGFP and SOX9 were a kind gift from Dr. Thierry Passeron [29].

\section{SOX9 transient transfection}

Empty vector or vector containing murine sox9 (a kind gift from Prof. Lukas Sommer) was transfected into B16F1 cells with jetPEI (Polyplus, France) according to manufacturer's protocol. Protein was isolated at $24 \mathrm{~h}$, $72 \mathrm{~h}, 144 \mathrm{~h}$, and $288 \mathrm{~h}$ after transfection and analyzed for sox 9 expression by western blot.

\section{Microarray and pathway analysis}

Gene expression of eGFP and SOX9 transfected cells were analyzed using the Affymetrix Human Genome U133 Plus 2.0 Array at the Functional Genomics Center Zurich (FGCZ). Differential gene expression was determined by $\mathrm{R}$ package limma [53]. Pathway analysis was performed using MetaCore (GeneGo Inc., New York, USA).

\section{Proliferation and cell cycle analysis}

For cell cycle analysis, the Click-iT EdU Alexa Fluor 647 Flow Cytometry Assay Kit (Invitrogen) was used. Cells were labelled with PI according to the manufacturer's protocol and the DNA content was measured using a BD FACSCanto II flow cytometer (BD Biosciences) and BD FACSDiva software (BD Biosciences). Cell cycle analysis was performed in triplicate.

\section{Boyden chamber invasion assay}

Cells were seeded on FluoroBlok 24-multiwell Insert System (351157, BD Biosciences) and Biocoat Tumor Invasion System (354165, BD Biosciences). The invasion assay was performed as per manufacturer's instructions. Migrated and invaded cells were labeled with Calcein AM fluorescent dye (354216, BD Biosciences) and fluorescence was measured with Tecan GENios (Tecan, Männendorf, Switzerland) using $485 \mathrm{~nm}$ excitation and $535 \mathrm{~nm}$ emission. Relative invasion was calculated as the ratio of the fluorescence of invading cells of the Biocoat Tumor Invasion System divided by the fluorescence of migrating cells of the FluoroBlok 24-multiwell Insert System. Boyden chamber assays were performed in triplicate.

\section{Viability assay}

Cells were seeded in 24-well microplates at a density of $2 \times 10^{4}$ cells, and cell growth was determined with a standard colorimetric assay measuring 3-(4,5-dimethylthiazol-2-yl)-2,5 diphenyltetrazolium bromide (MTT) (Sigma-Aldrich, St Louis, MO, USA) reactivity after $72 \mathrm{~h}$. MTT assays were performed in triplicate.

\section{Chromatin immunoprecipitation}

ChIP analysis was performed as previously described [54]. The Sox9 antibody was from Santa Cruz Biotechnology (sc-20095, Santa Cruz Biotechnology). Primer sequences were designed around SOX9 binding motifs $[55,56]$ from the transcription start site (TSS) to $3 \mathrm{~KB}$ upstream of the TSS. Primers for p21, COL2A1, TMEM158, TBX3, FYB, SOX10, and IP10 are shown in Table 4.

\section{In vivo metastasis}

B16F1 cells were transfected with empty vector or vector containing murine sox9. $2 \times 10^{5}$ cells were injected intravenously into C57BL/6 J mice, five mice per group (Harlan Laboratories). After 12 days mice were sacrificed and lungs were examined for metastasis. Statistics were performed using the Mann-Whitney U test. All animal experiments have been approved by the veterinary authorities of Canton

Table 4 Primers for ChIP

\begin{tabular}{ll}
\hline Genes & Primer \\
\hline COL2A1-F & ATCCTCCTTTGTGAGGCTTGTT \\
COL2A1-R & AGTACGAGAGAACCCACTGGAC \\
p21-F & TGATGTGCCACAGTTCACAA \\
p21-R & TCCTGCCAGTTTCCTGTTC \\
TMEM158-F & TCTGCTGTGTTGGAGCCATT \\
TMEM158-R & GTCTCGCCTTAGTGCTACCG \\
TBX3-F & CTCGCCCCTTTCTTCCCTT \\
TBX3-R & GGGGGTGTTATGAGCCAACA \\
FYB-F & CTCACATTGCATGGGGACG \\
FYB-R & ATGGGCTTATCACCGGAAGG \\
SOX10-F & CCTCTGCCTCGTGTGACTAC \\
SOX10-R & TCCTGTCTGGAGTGGGCTG- \\
IP10-F & GGGAAATCCGTAACTTGGA \\
IP10-R & AAGCCATTITCCCTCCCTAA \\
\hline
\end{tabular}


of Zurich, Switzerland, and were performed in accordance with Swiss law.

\section{TCGA analysis}

The SKCM DNA methylation, RNA-seq, and clinical dataset were downloaded on 28 July 2014 for analysis. Normalized reads from the level 3 RNA-seq data were used for analysis. The dataset was segregated into primary tumors and metastatic tumors for analysis. Chi-squared test was performed on the clinical parameters between the SOX9 high and low groups. Differential expression was analyzed with voom from the limma package [53]. Log rank test and Cox proportional hazard ratio were analyzed by the survival R package [57]. DNA methylation $\beta$-values were calculated by minfi [58]. Correlation was calculated by Spearman's rank correlation coefficient.

\section{Additional files}

\section{Additional file 1: Figure S1. MLANA expression between the} proliferative and invasive phenotype. Ten melanoma cell cultures were divided into the proliferative and invasive phenotype by expression of MLANA. GAPDH was used as loading control.

Additional file 2: Table S1. 73 genes with anti-correlative methylation and gene expression. Analysis of the methylation and expression profiles of the ten melanoma cell cultures produced this list of significant genes with a peak score greater than 2 from the MeDIP methylation array and with a fold change greater than 1 from the gene expression microarray.

Additional file 3: Table S2. Pathway analysis of 73 gene signature. Genes were uploaded to Metacore and analyzed for significant pathway enrichment in GO Processes, Process Networks and Pathway Maps.

Additional file 4: Table S3. Differential gene analysis of SOX9 overexpression. Limma output table for differentially expressed genes between SOX9 overexpression and control M010817 melanoma cells.

Additional file 5: Table S4. Clinical parameters of primary melanoma patients. TNM stage, gender, and tumor location between the SOX9 high and SOX9 low patients were evaluated by the Chi-squared test. Age was evaluated by Student's t-test. * represents $p<0.05$.

Additional file 6: Table S5. Differential gene analysis of SOX9 high and low primary melanoma patients. Limma output table for SOX9 high primary melanoma patients versus SOX9 low primary melanoma patients.

Additional file 7: Table S6. Differential gene analysis of SOX9 high and low metastatic melanoma patients. Limma output table for SOX9 high metastatic melanoma patients versus SOX9 low metastatic melanoma patients.

Additional file 8: Table S7. Overlap of SOX9 overexpression and metastatic melanoma patients. List of genes overlapping from M010817 melanoma cells overexpressing SOX9 and SOX9 high metastatic melanoma patients.

\section{Competing interests}

The authors declare that they have no competing interests.

\section{Authors' contributions}

PFC, OS, DSW, DZ, SCF, BB, MIGR, OME, and SMG performed the experiments. PFC, OS, DSW, DZ, and SCF analyzed the data. PFC, RS, RD, and MPL contributed to the overall design of the study and directed research. PFC, $\mathrm{SH}, \mathrm{LS}, \mathrm{RD}$, and MPL drafted the manuscript. All authors read and approved the final manuscript.

\section{Acknowledgements}

This work was financially supported by the Georg und Bertha Schwyzer-Winiker Stiftung (RD), the Forschungskredit FK-13-042 (PFC), the Swiss Cancer League and the Swiss National Science Foundation (LS, MPL), the Promedica Foundation (OS), and the Verein für Hautkrebsforschung (MPL). The University Research Priority Program (URPP) in translational cancer research at the University of Zürich provided cell lines used in this work. We thank the Cancer Biology PhD Program of the University/ETH Zürich for support and advice. We would also like to thank The Cancer Genome Atlas (TCGA) for providing important clinical and genomic data. Finally, all research on human materials was done according to Swiss law and following the Declaration of Helsinki on medical research. Consenting patients contributed surplus melanoma biopsy material according to IRB approval numbers (Ek.647/800), and cell lines were generated following IRB KEK-ZH-Nr.2014-0425.

\section{Author details}

'Department of Dermatology, Faculty of Medicine, University Hospital Zürich, and University of Zürich, Wagistrasse 14, CH-8952 Zürich, Switzerland.

${ }^{2}$ Faculty of Mathematics and Natural Sciences, Institute of Molecular Life Sciences, University of Zürich, Zürich, Switzerland. ${ }^{3}$ Cell and Developmental Biology, Institute of Anatomy, University of Zürich, Zürich, Switzerland. ${ }^{4}$ Institute of Veterinary Biochemistry and Molecular Biology, University of Zürich, Zürich, Switzerland.

Received: 14 October 2014 Accepted: 23 January 2015

Published online: 22 February 2015

\section{References}

1. Dupin $E$, Le Douarin NM. Development of melanocyte precursors from the vertebrate neural crest. Oncogene. 2003;22:3016-23.

2. Garbe C, Leiter U. Melanoma epidemiology and trends. Clin Dermatol. 2009;27:3-9.

3. MacKie RM, Hauschild A, Eggermont AM. Epidemiology of invasive cutaneous melanoma. Ann Oncol. 2009;20:vi1-7.

4. Quintana E, Shackleton M, Foster HR, Fullen DR, Sabel MS, Johnson TM, et al. Phenotypic heterogeneity among tumorigenic melanoma cells from patients that is reversible and not hierarchically organized. Cancer Cell. 2010;18:510-23.

5. Perego $M$, Tortoreto $M$, Tragni $G$, Mariani L, Deho $P$, Carbone $A$, et al. Heterogeneous phenotype of human melanoma cells with in vitro and in vivo features of tumor-initiating cells. J Invest Dermatol. 2010;130:1877-86.

6. Roesch A, Fukunaga-Kalabis M, Schmidt EC, Zabierowski SE, Brafford PA, Vultur A, et al. A temporarily distinct subpopulation of slow-cycling melanoma cells is required for continuous tumor growth. Cell. 2010;141:583-94.

7. Landsberg J, Kohlmeyer J, Renn M, Bald T, Rogava M, Cron M, et al. Melanomas resist T-cell therapy through inflammation-induced reversible dedifferentiation. Nature. 2012;490:412-6.

8. Romano E, Schwartz GK, Chapman PB, Wolchock JD, Carvajal RD. Treatment implications of the emerging molecular classification system for melanoma. Lancet Oncol. 2011;12:913-22.

9. Hoek KS, Goding CR. Cancer stem cells versus phenotype-switching in melanoma. Pigment Cell Melanoma Res. 2010;23:746-59.

10. Freedman JA, Tyler DS, Nevins JR, Augustine CK. Use of gene expression and pathway signatures to characterize the complexity of human melanoma. Am J Pathol. 2011;178:2513-22.

11. Hoek KS, Schlegel NC, Brafford P, Sucker A, Ugurel S, Kumar R, et al. Metastatic potential of melanomas defined by specific gene expression profiles with no BRAF signature. Pigment Cell Res. 2006;19:290-302.

12. Hendrix MJ, Seftor EA, Seftor RE, Kasemeier-Kulesa J, Kulesa PM, Postovit LM. Reprogramming metastatic tumour cells with embryonic microenvironments. Nat Rev Cancer. 2007:7:246-55.

13. Widmer DS, Cheng PF, Eichhoff OM, Belloni BC, Zipser MC, Schlegel NC, et al. Systematic classification of melanoma cells by phenotype-specific gene expression mapping. Pigment Cell Melanoma Res. 2012;25:343-53.

14. Eichhoff OM, Zipser MC, Xu M, Weeraratna AT, Mihic D, Dummer R, et al. The immunohistochemistry of invasive and proliferative phenotype switching in melanoma: a case report. Melanoma Res. 2010;20:349-55.

15. Widmer DS, Hoek KS, Cheng PF, Eichhoff OM, Biedermann T, Raaijmakers $\mathrm{MI}$, et al. Hypoxia contributes to melanoma heterogeneity by triggering HIF1alpha-dependent phenotype switching. J Invest Dermatol. 2013;133:2436-43

16. Carreira S, Goodall J, Denat L, Rodriguez M, Nuciforo P, Hoek KS, et al. Mitf regulation of Dia1 controls melanoma proliferation and invasiveness. Genes Dev. 2006;20:3426-39. 
17. Cheli Y, Giuliano S, Botton T, Rocchi S, Hofman V, Hofman P, et al. Mitf is the key molecular switch between mouse or human melanoma initiating cells and their differentiated progeny. Oncogene. 2011;30:2307-18.

18. Goodall J, Carreira S, Denat L, Kobi D, Davidson I, Nuciforo P, et al. Brn-2 represses microphthalmia-associated transcription factor expression and marks a distinct subpopulation of microphthalmia-associated transcription factor-negative melanoma cells. Cancer Res. 2008;68:7788-94.

19. Plass C. Cancer epigenomics. Hum Mol Genet. 2002;11:2479-88.

20. Baylin SB, Jones PA. A decade of exploring the cancer epigenome - biological and translational implications. Nat Rev Cancer. 2011;11:726-34.

21. Hansen KD, Timp W, Bravo HC, Sabunciyan S, Langmead B, McDonald OG, et al. Increased methylation variation in epigenetic domains across cancer types. Nat Genet. 2011;43:768-75.

22. Molognoni F, Cruz AT, Meliso FM, Morais AS, Souza CF, Xander P, et al. Epigenetic reprogramming as a key contributor to melanocyte malignant transformation. Epigenetics. 2011;6:450-64.

23. Sigalotti L, Coral S, Nardi G, Spessotto A, Cortini E, Cattarossi I, et al. Promoter methylation controls the expression of MAGE2, 3 and 4 genes in human cutaneous melanoma. J Immunother. 2002;25:16-26.

24. Nguyen T, Kuo C, Nicholl MB, Sim MS, Turner RR, Morton DL, et al. Downregulation of microRNA-29c is associated with hypermethylation of tumor-related genes and disease outcome in cutaneous melanoma. Epigenetics. 2011:6:388-94.

25. Deng T, Kuang Y, Wang L, Li J, Wang Z, Fei J. An essential role for DNA methyltransferase $3 a$ in melanoma tumorigenesis. Biochem Biophys Res Commun. 2009;387:611-6.

26. Schinke C, Mo Y, Yu Y, Amiri K, Sosman J, Greally J, et al. Aberrant DNA methylation in malignant melanoma. Melanoma Res. 2010;20:253-65.

27. Alcazar O, Achberger S, Aldrich W, Hu Z, Negrotto S, Saunthararajah Y, et al. Epigenetic regulation by decitabine of melanoma differentiation in vitro and in vivo. Int J Cancer. 2012;131:18-29.

28. Cheung M, Briscoe J. Neural crest development is regulated by the transcription factor Sox9. Development. 2003;130:5681-93.

29. Passeron T, Valencia JC, Namiki T, Vieira WD, Passeron H, Miyamura $Y$, et al. Upregulation of SOX9 inhibits the growth of human and mouse melanomas and restores their sensitivity to retinoic acid. J Clin Invest. 2009;119:954-63.

30. Hoek KS, Eichhoff OM, Schlegel NC, Dobbeling U, Kobert N, Schaerer L, et al. In vivo switching of human melanoma cells between proliferative and invasive states. Cancer Res. 2008:68:650-6.

31. Weber M, Davies JJ, Wittig D, Oakeley EJ, Haase M, Lam WL, et al. Chromosome-wide and promoter-specific analyses identify sites of differential DNA methylation in normal and transformed human cells. Nat Genet. 2005;37:853-62.

32. Smyth GK. Linear models and empirical bayes methods for assessing differential expression in microarray experiments. Stat Appl Genet Mol Biol. 2004;3:Article3.

33. Passeron T, Valencia JC, Bertolotto C, Hoashi T, Le Pape E, Takahashi K, et al SOX9 is a key player in ultraviolet B-induced melanocyte differentiation and pigmentation. Proc Natl Acad Sci U S A. 2007;104:13984-9.

34. Cook AL, Smith AG, Smit DJ, Leonard JH, Sturm RA. Co-expression of SOX9 and SOX10 during melanocytic differentiation in vitro. Exp Cell Res. 2005;308:222-35

35. Eichhoff OM, Weeraratna A, Zipser MC, Denat L, Widmer DS, Xu M, et al. Differential LEF1 and TCF4 expression is involved in melanoma cell phenotype switching. Pigment Cell Melanoma Res. 2011;24:631-42.

36. Bell DM, Leung KK, Wheatley SC, Ng L, Zhou S, Ling KW, et al. SOX9 directly regulates the type-II collagen gene. Nat Genet. 1997;16:174-8.

37. Shakhova O, Cheng P, Mishra PJ, Zingg D, Schaefer SM, Debbache J, et al. Antagonistic cross-regulation between Sox9 and Sox 10 controls an anti-tumorigenic program in Melanoma. PLoS Genet. 2015;11:e1004877.

38. Mihic-Probst D, Ikenberg K, Tinguely M, Schraml P, Behnke S, Seifert B, et al. Tumor cell plasticity and angiogenesis in human melanomas. PLoS One. 2012; 7:e33571.

39. Hendrix MJ, Seftor EA, Hess AR, Seftor RE. Molecular plasticity of human melanoma cells. Oncogene. 2003;22:3070-5.

40. Zipser MC, Eichhoff OM, Widmer DS, Schlegel NC, Schoenewolf NL, Stuart D, et al. A proliferative melanoma cell phenotype is responsive to RAF/MEK inhibition independent of BRAF mutation status. Pigment Cell Melanoma Res. 2011:24:326-33.
41. Fraga MF, Herranz M, Espada J, Ballestar E, Paz MF, Ropero S, et al. A mouse skin multistage carcinogenesis model reflects the aberrant DNA methylation patterns of human tumors. Cancer Res. 2004;64:5527-34

42. Bonazzi VF, Nancarrow DJ, Stark MS, Moser RJ, Boyle GM, Aoude LG, et al. Cross-platform array screening identifies COL1A2, THBS1, TNFRSF10D and UCHL1 as genes frequently silenced by methylation in melanoma. PLoS One. 2011;6:e26121.

43. Koga Y, Pelizzola M, Cheng E, Krauthammer M, Sznol M, Ariyan S, et al. Genome-wide screen of promoter methylation identifies novel markers in melanoma. Genome Res. 2009;19:1462-70.

44. Muthusamy V, Duraisamy S, Bradbury CM, Hobbs C, Curley DP, Nelson B, et al. Epigenetic silencing of novel tumor suppressors in malignant melanoma. Cancer Res. 2006:66:11187-93.

45. Conway K, Edmiston SN, Khondker ZS, Groben PA, Zhou X, Chu H, et al. DNA-methylation profiling distinguishes malignant melanomas from benign nevi. Pigment Cell Melanoma Res. 2011;24:352-60.

46. Lian CG, Xu Y, Ceol C, Wu F, Larson A, Dresser K, et al. Loss of 5 -hydroxymethylcytosine is an epigenetic hallmark of melanoma. Cell. 2012;150:1135-46.

47. Rajasekaran K, Kumar P, Schuldt KM, Peterson EJ, Vanhaesebroeck B, Dixit V et al. Signaling by Fyn-ADAP via the Carma1-BCl-10-MAP3K7 signalosome exclusively regulates inflammatory cytokine production in NK cells. Nat Immunol. 2013;14:1127-36

48. Peres J, Prince S. The T-box transcription factor, TBX3, is sufficient to promote melanoma formation and invasion. Mol Cancer. 2013;12:117.

49. Rodriguez M, Aladowicz E, Lanfrancone L, Goding CR. Tbx3 represses E-cadherin expression and enhances melanoma invasiveness. Cancer Res. 2008;68:7872-81.

50. Raaijmakers MIG, Widmer DS, Maudrich M, Koch T, Langer A, Flace A, et al. A new live cell biobank workflow efficiently recovers heterogeneous melanoma cells from native biopsies. Exp Dermatol. 2015.

51. Geertsen RC, Hofbauer GF, Yue FY, Manolio S, Burg G, Dummer R. Higher frequency of selective losses of HLA-A and -B allospecificities in metastasis than in primary melanoma lesions. J Invest Dermatol. 1998;111:497-502.

52. Bock C, Reither S, Mikeska T, Paulsen M, Walter J, Lengauer T. BiQ Analyzer: visualization and quality control for DNA methylation data from bisulfite sequencing. Bioinformatics. 2005;21:4067-8.

53. Smyth GK. Limma: linear models for microarray data. New York: Springer; 2005

54. Santoro R. Analysis of chromatin composition of repetitive sequences: the ChIP-Chop assay. Methods Mol Biol. 2014;1094:319-28.

55. Kadaja M, Keyes BE, Lin M, Pasolli HA, Genander M, Polak L, et al. SOX9: a stem cell transcriptional regulator of secreted niche signaling factors. Genes Dev. 2014;28:328-41.

56. Mathelier A, Zhao X, Zhang AW, Parcy F, Worsley-Hunt R, Arenillas DJ, et al. JASPAR 2014: an extensively expanded and updated open-access database of transcription factor binding profiles. Nucleic Acids Res. 2014;42:D142-7.

57. Therneau TM: A Package for Survival Analysis in S. In R package version 237-4; 2013.

58. Aryee MJ, Jaffe AE, Corrada-Bravo H, Ladd-Acosta C, Feinberg AP, Hansen $K D$, et al. Minfi: a flexible and comprehensive Bioconductor package for the analysis of Infinium DNA methylation microarrays. Bioinformatics. 2014;30:1363-9.

\section{Submit your next manuscript to BioMed Central and take full advantage of:}

- Convenient online submission

- Thorough peer review

- No space constraints or color figure charges

- Immediate publication on acceptance

- Inclusion in PubMed, CAS, Scopus and Google Scholar

- Research which is freely available for redistribution 